\title{
Development of the First Force-Controlled Robot for Otoneurosurgery
}

Philipp A. Federspil, MD; Urban W. Geisthoff, MD; Dominik Henrich, PhD; Peter K. Plinkert, MD, PhD

Objective: In some surgical specialties (eg, orthopedics), robots are already used in the operating room for bony milling work. Otological surgery and otoneurosurgery may also greatly benefit from the enhanced precision of robotics. Study Design: Experimental study on robotic milling of oak wood and human temporal bone specimen. Methods: A standard industrial robot with a six-degrees-of-freedom serial kinematics was used, with force feedback to proportionally control the robot speed. Different milling modes and characteristic path parameters were evaluated to generate milling paths based on computeraided design (CAD) geometry data of a cochlear implant and an implantable hearing system. Results: The best-suited strategy proved to be the spiral horizontal milling mode with the burr held perpendicular to the temporal bone surface. To reduce groove height, the distance between paths should equal half the radius of the cutting burr head. Because of the vibration of the robot's own motors, a high oscillation of the SD of forces was encountered. This oscillation dropped drastically to nearly 0 Newton $(N)$ when the burr head made contact with the dura mater, because of its damping characteristics. The cutting burr could be kept in contact with the dura mater for an extended period without damaging it, because of the burr's blunt head form. The robot moved the burr smoothly according to the encountered resistances. Conclusion: The study reports the first development of a functional robotic milling procedure for otoneurosurgery with force-based speed control. Future plans include implementation of ultrasound-based local navigation and performance of robotic mastoidectomy. Key Words: Robotics, implant, mastoid, mastoidectomy, otorhinolaryngological surgery, reaming. Laryngoscope, 113:465-471, 2003

From the Department of Otorhinolaryngology, Head \& Neck Surgery (P.A.F., U.G., P.K.P.), University of Saarland, Kirrberger Strasse, Homburg (Saar), Germany; and the Robotics and Embedded Systems (RESY) (D.H.), Informatics Faculty, University of Kaiserslautern, Kaiserslautern, Germany

Supported by the Deutsche Forschungsgemeinschaft (DFG) in the special research cluster SPP 1124, "Medical Navigation and Robotics" (grant PL 136/5-1) 8,2002 .

Editor's Note: This Manuscript was accepted for publication October

Send Correspondence to Philipp A. Federspil, MD, Klinik und Poliklinik für Hals-Nasen-Ohrenheilkunde, Universitätskliniken des Saarlandes, Kirrberger Strasse, D-66421 Homburg (Saar), Germany. E-mail: Ph.Federspil@uniklinik-saarland.de

\section{INTRODUCTION}

The robot that serves the surgeon today is different from its anthropomorphic counterparts in science fiction movies such as "Star Wars." It resembles more closely an industrial robot for automobile assembly; today's surgical robots are actually derived from this technology. ${ }^{1-3}$ The role of robots in surgery is small but still important; they merely create holes for total hip replacement. However, these holes are much more accurately drilled than those that could be made by a human being. Currently, there are three robots on the market, with most of them used in German operating rooms: ROBODOC (Integrated Surgical Systems Inc., Davis, CA) and CASPAR (developed by ortoMAQUET Inc., Rastatt, Germany, and currently marketed by URS Ortho Inc., Rastatt, Germany) are used in orthopedic surgery for total hip and knee replacement, whereas EVOLUTION 1 (URS Inc., Schwerin, Germany) is currently approved by the European Community for robotic neuroendoscopy. These systems are, strictly speaking, robots because they are freely programmable for a complete surgical step, and are not to be confused with (tele)manipulators such as ZEUS (ComputerMotion Inc.) or DAVINCI (Intuitive Surgical Inc.), which act exclusively under direct control of the surgeon in a masterslave fashion. ${ }^{2,3}$

Otoneurosurgery may greatly benefit from enhanced precision through robotics or from new procedures only made possible with robotic aid. ${ }^{4}$ There are a number of different implants in otological surgery and otoneurosurgery (e.g., cochlear implants and implantable hearing systems), which require a cavity in the calvarial bone for the implanted main module. Taking into account that 90 million people worldwide have hearing loss, more implants of this type are likely to be developed in the future. The geometry of such an implant is simple; therefore, this seems to be a good application for investigating robotic milling. Although today the cavity for an implant is created by the surgeon personally, this procedure is timeconsuming and leaves the surgeon with macroscopic milling work before the much more important microsurgical fine work. As a result, surgeons may become tired and/or experience boredom during the milling of the implant bed, leaving them with an increased tendency for tremor and loss of concentration. Therefore, the robot would improve quality of the procedure twofold: first, by improving accu- 

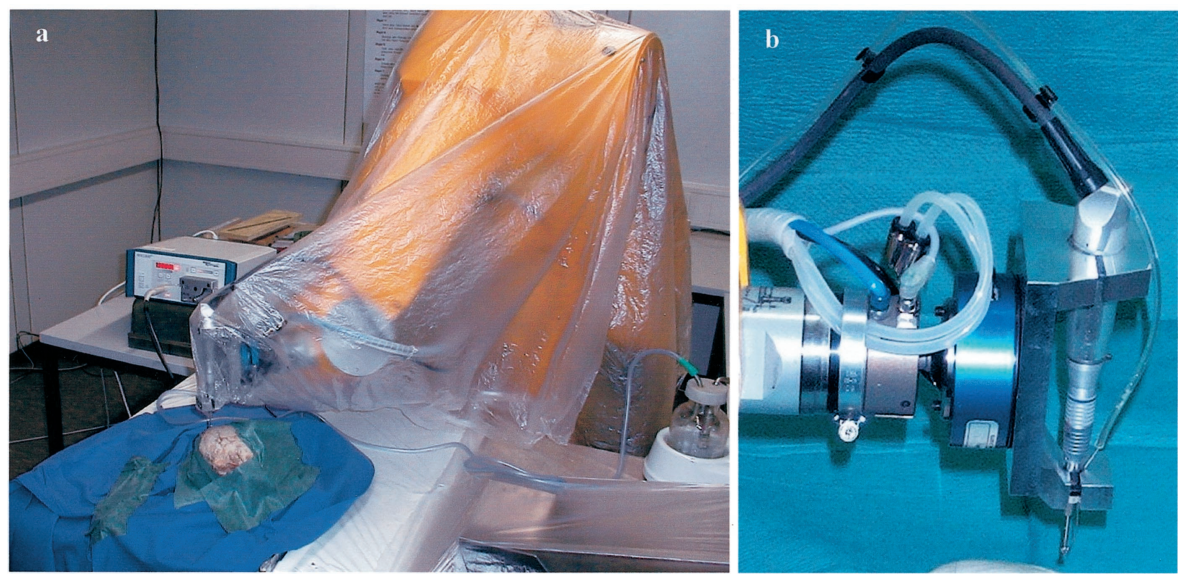

Fig. 1. (A) Experimental setup with the Stäubli RX 130 covered with transparent sheet for protection, the temporal bone specimen, and the Aesculap Microspeed electrical burr (left) including irrigation and suction (right). (B) End of robot arm equipped with pneumatic collision protection, a force/torque sensor, and the tight coupling of the milling burr with the saline irrigation line. racy of the implant bed itself and, second, by leaving surgeons concentrated and fresh for their demanding work. When this has been accomplished, development of a robot for automated milling of the complete mastoid is planned. ${ }^{5}$ The mastoidectomy was chosen not only because it is a common procedure as well as a stand-alone task, but also because the entrance to the lateral skull base has many applications.

\section{MATERIALS AND METHODS}

\section{Robot, Robot Control, and Sensors}

The RX-130 (Stäubli Inc.) robot with serial kinematics and 6 degrees of freedom (Fig. 1A) has a relative repositioning accuracy of $0.3 \mathrm{~mm}$. It is the same type of robot as CASPAR. The robot was computer controlled (operating system V+, Adept CS7 VME controller). The force and torque sensor was a KMS 90M31 sensor (JR-3 Inc., Woodland, CA) with a measurement range up to 63 Newton $(\mathrm{N})$ and a sampling rate of $400 \mathrm{~Hz}$, and was placed between the end of the robot arm and the coupling device for the burr (Fig. 1B). The absolute values and SD of the forces were calculated online in samples of 20 consecutive measurements. The maximum for the absolute value of the force was set to $10 \mathrm{~N}$, in accordance with previous research. ${ }^{5}$ Force levels greater than $40 \mathrm{~N}$ caused abortion of the robot's action. In addition, the robot possessed a built-in safety feature, consisting of a mechanical breakaway.

\section{Burr}

We used the Microspeed EC Electric motor system (Aesculap Inc., Tuttlingen, Germany), allowing for rotation ranging from 10,000 to $30,000 \mathrm{rpm}$ (low-speed motor GD657) or 10,000 to 75,000 rpm (high-speed motor GD656); the setup included an integrated cooling system (Fig. 1A and B). Cutting burrs and diamond burrs with diameters ranging from 1.3 to $4.5 \mathrm{~mm}$ were used. The burr was mounted on an individually designed aluminum block that was connected to the force/torque sensor, which was mounted on the robot's wrist. This connection was made as tight as possible to prevent loss of high-frequency vibration for
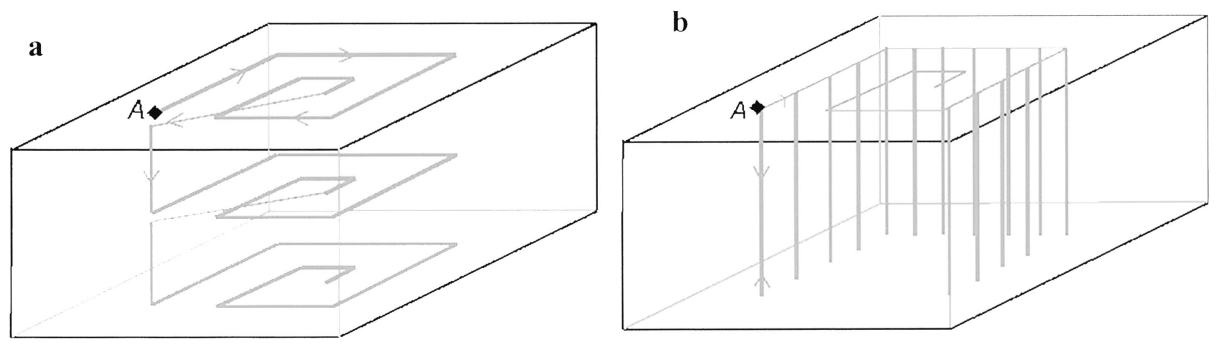

c

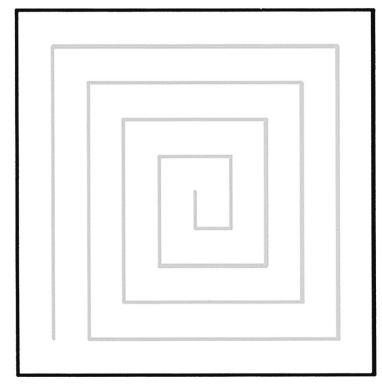

d

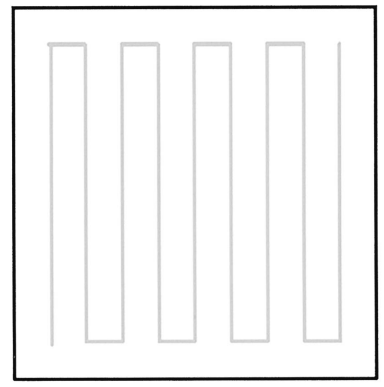

Fig. 2. Different modes of milling. (A) Horizontal milling, (B) vertical milling, (C) spiral horizontal milling, and (D) meandering horizontal milling (explained in text). $A$ is the starting point. 
a

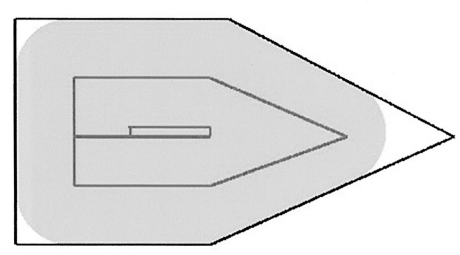

b

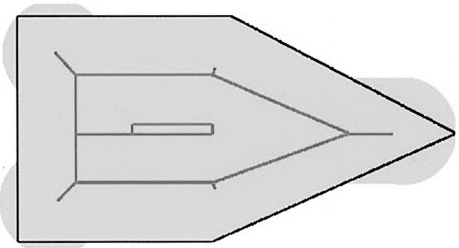

Fig. 3. View of the internal contour of a given volume. (A) The internal contour is never overstepped; therefore, the corners are not fully drilled out. (B) The internal contour is overstepped in the corners; therefore, a given object fits inside (explained in text).

the sensor control (Fig. 1B). The burr was always held perpendicular to the temporal bone surface.

\section{Milling Modes}

The following modes of moving the robot's burr were evaluated on oak wood:

1. Vertical milling mode (Fig. 2B). One hole was milled completely to the ground; then the robot moved the burr one step horizontally to the next drilling hole.

2. Horizontal milling mode (Fig. 2A). The robot moved the burr in a horizontal plane, then stepped deeper to next plane underneath.

a. Spiral horizontal milling mode (Fig. 2C). The burr was moved in a spiral fashion within one horizontal plane, either from outside to inside or vice versa.

b. Meander horizontal milling mode (Fig. 2D). The burr was moved in a meandering fashion within one horizontal plane.

\section{Milling Strategy With Respect to Contour}

The required cavity volume for the milling task and creation of paths was taken from original computer-aided design data of the cochlear implant by Med-El, Inc., and the totally implantable hearing system TICA (formerly marketed by Implex, Inc.). Because of the geometry of the burr, which is spherical in this setting, there are two strategies for performing the milling task. In the first scenario, the borders of the given volume (the contour) are respected absolutely and never overstepped by the burr, which may leave remnants of bone in the corners (Fig. 3A). An object will probably not fit in the resulting cavity, but this strategy is important for preserving noble structures when performing mastoidectomy. In the second situation, the borders are not always respected or are overstepped purposely to remove bone from the corners, to allow the object to fit into the cavity (Fig. 3B). The two strategies are equivalent when the diameter of the burr becomes infinitely small, but this gain in accuracy is coupled with the increased time needed for the task with a smaller burr.
Changing the diameter or the design of the burr during the milling task may be a means to overcome this problem.

\section{Path Parameters}

The following path parameters were defined, as partially shown in Figure 4:

1. $r_{1}$ is the distance between the outmost contour and the center of the burr on the first path in the horizontal milling mode.

2. $r_{2}$ is the distance between the outmost contour and the center of the burr on the first path in the vertical milling mode (equivalent to $r_{1}$ ).

3. $r_{3}$ is the distance the burr is moved centrally to the next path.

4. $r_{4}$ is the distance the burr is moved downward to the next plane in the horizontal milling mode.

Parameter $r_{1}$ is equivalent to the radius of the burr. Parameter $r_{3}$ alternated between $0.5 \times r_{1}, 0.7 \times r_{1}$, and 1.0x $r_{1}$ whereas $r_{4}$ was $1.06,1.26$, or $1.50 \mathrm{~mm}$. The robot was registered to the specimen (see "Specimen") by defining three points in space at which the robot was positioned. The robot was moved manually to these points with the aid of a zero-force control. While measuring the force on the sensor, the computer generated a movement of the robot in the direction of the force, thereby reducing the force to $0 \mathrm{~N}$. The movement was calculated proportionally to the measured force (Fig. 5). The three points define the first plane of the milling task. In a later phase of the experiments, we implemented this proportional rule for sensory feedback control of the robot's movements by extracting the force levels in the very direction of the robot feed, averaging a sample of seven measurements.

\section{Specimen}

The milling tasks were performed on the following specimens:
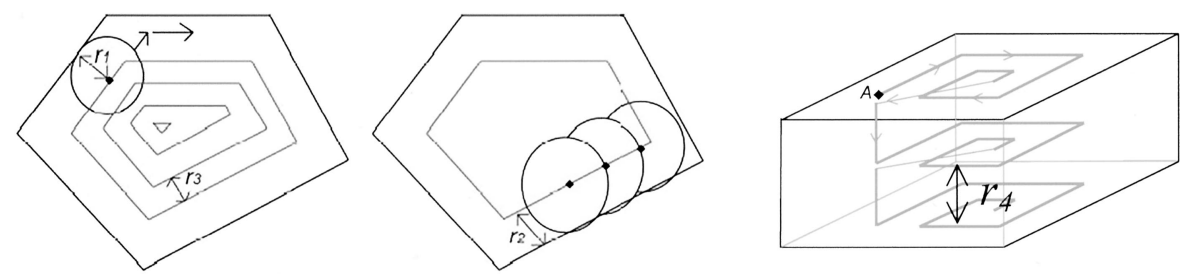

Fig. 4. Various path parameters. The path $r_{1}$ is the distance between the outmost contour and the center of the burr on the first path in the horizontal milling mode, $r_{3}$ is the distance the burr is moved centrally in the spiral horizontal milling mode, $r_{2}$ is the distance between the outmost contour and the center of the burr on the first path in the vertical milling mode (equivalent to $r_{1}$ ), and $r_{4}$ is the distance the burr moves downward to the next plane in the horizontal milling mode. 


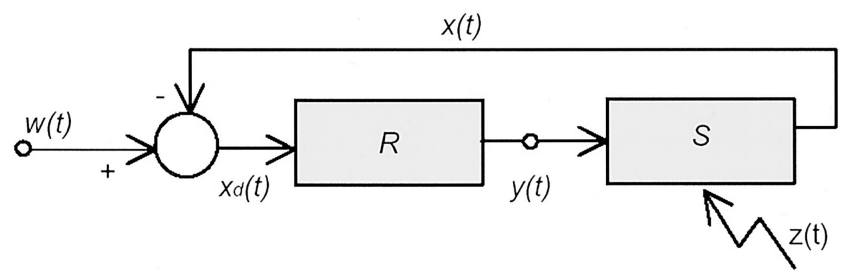

Fig. 5. Zero-force control. The robot arm is moved proportionally to the measured forces while maintaining a force of $0 \mathrm{~N}$. Thereby, the robot arm can be easily moved manually from one point to another by the surgeon. $\mathrm{R}$, robot; $\mathrm{S}$, sensor; $\mathrm{x}(\mathrm{t})$, force exerted on the robot arm; $w(t)$, regulating force (zero in our case); $x d(t)$, difference between force exerted and regulated; $y(t)$ and $z(t)$, influencing factors.

1. Oak wood. Oak is recommended by the burr manufacturer as an adequate material for testing new milling parameters.

2. Formaldehyde-preserved human temporal bones. The specimens were taken from donors who had donated their bodies to science, with informed consent prior to death. Beforehand, the study was approved by the Ethics Commission of Saarland (Saarbrücken, Germany).

\section{RESULTS}

The positioning of the robot arm by means of zeroforce control was easy to perform. However, it was difficult to exactly define an adequately horizontal (or tangential) plane to the temporal bone specimen and to position the robot near enough to the surface. When the burr was too far away, it moved too long of a time in the actual paths designated for milling without touching the surface. However, when the robot was positioned close to the surface, collision with the specimen sometimes occurred, with the sensor giving way for safety reasons.

The paths for different milling modes were calculated offline by a computer using our own algorithm. Because the milling task was to fit an implant into a cavity in the temporal bone, the milling strategy was chosen to (minimally) overstep the contour in selected areas (Fig. 3B).

When different milling modes were used on a piece of oak, the time required in the vertical milling mode was more than double that in the spiral horizontal milling mode, even for $r_{3}$ (the distance in between paths) equal to $r_{1}$ (Fig. 6). In the spiral horizontal milling mode, the increase in time needed to complete the milling task when changing $r_{3}$ from $1.0 \mathrm{x} r_{1}$ to $0.7 \mathrm{x} r_{1}$ and $0.5 \mathrm{x} r_{1}$ was relatively small; by halving the distance between paths (i.e., doubling the number of paths), the time for the milling task increased from 181 to 250 seconds, or by only $38 \%$. However, the increase in required time for the vertical milling mode was unproportionally high (Fig. 6). The larger the distance between paths, the higher are the grooves remaining at the bottom of the cavity (Fig. 7). When $r_{3}$ equaled $1.0 \mathrm{x} r_{1}$, the grooves left behind were 0.85 $\mathrm{mm}$, whereas with half the path distance they were only $0.4 \mathrm{~mm}$, so the surface was much smoother. The vertical milling mode proved to be inadequate because the time needed for milling was much longer and cooling was not as easily manageable as in the horizontal milling mode.

The forces were measured online with a sampling rate of $400 \mathrm{~Hz}$. The SD of the force levels within the $\mathrm{x}$ component

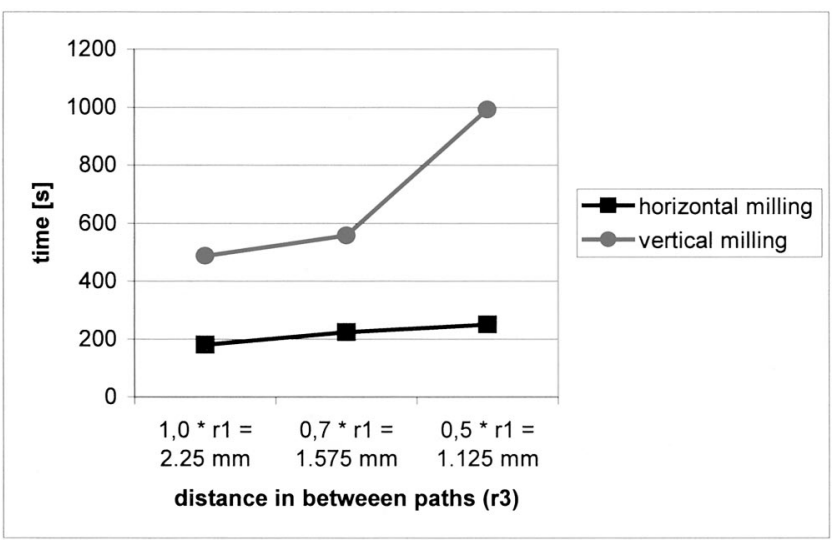

Fig. 6. Time to complete the milling task while varying the distance between paths as a function of the distance between the internal contour and the center of the burr on the first path (equivalent to the radius of the burr); comparison showing horizontal versus vertical milling mode.

turned out to be more interesting than the absolute values. Even when the robot was not moving and was not in contact with the specimen, the SD of the force did not equal $0 \mathrm{~N}$, but oscillated between 0 and $0.04 \mathrm{~N}$. With the burr rotating at $30,000 \mathrm{rpm}$, the SD oscillated between 0.02 and $0.22 \mathrm{~N}$ when the robot was not in contact with the specimen. However, the $\mathrm{SD}$ increased and oscillated to a much greater extent (up to

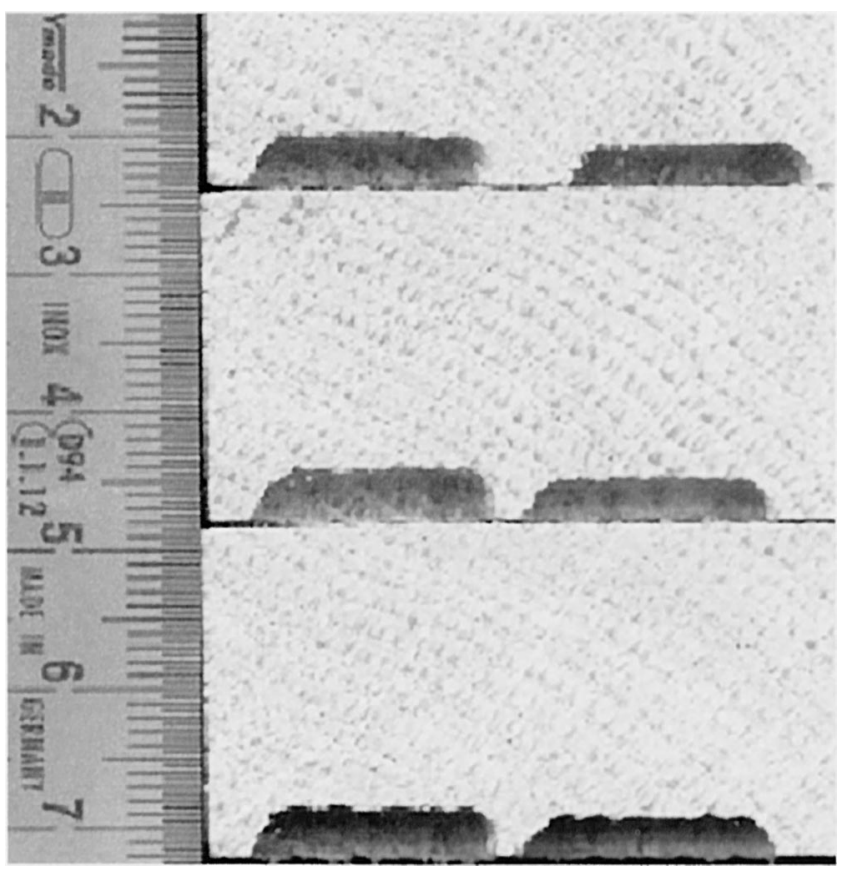

Fig. 7. Experimental milling of a piece of oak. A 4.5-mm-diameter cutting burr was used in all instances. The path $r_{1}$ was set to 2.25 $\mathrm{mm}$ (half the diameter) for horizontal milling (explained in text; see Fig. 2). The path $r_{2}$ was set to $2.0 \mathrm{~mm}$ for vertical milling. The left column was performed in the vertical milling mode, the right column in the spiral horizontal milling mode. With increasing distance between paths $r_{3}$ and distance between planes $r_{4}$ (only relevant in the horizontal milling mode), an increase in groove height is observable. Metric ruler is in centimeters. 
$1 \mathrm{~N}$, with peak values greater than $3 \mathrm{~N}$ ) as the robot performed the milling task. The rotation of the burr at 30,000 rpm did not contribute greatly to the variation of the SD (Fig. 8). However, after the last bony layer at the bottom of the implant bed had been removed by the burr and the burr was in contact with the dura mater, the SD decreased to almost $0 \mathrm{~N}$. Some peaks that were observed while the burr moved along the dura were due to remnants of bone. The burr could be moved for an extended period along the original milling path without damaging the dura. All milling work could be performed by the cutting burr, which works more quickly and produces less heat than the diamond burr (Fig. 9).

In our first investigation, the robot performed the milling task with a constant speed, whether it was still above the surface or within the bone. The resulting forces reached high values, often exceeding our empirically discovered limit ${ }^{5}$ of $10 \mathrm{~N}$, or even causing abortion of the robot's action by exceeding $40 \mathrm{~N}$. This could be counteracted by reducing the robot's working speed, but this in turn would increase the total execution time. In addition, the movements looked clumsy. This was especially true when the distance $r_{3}$ the burr is moved centrally to the next path was large, leaving relatively high grooves. With relentless movements of the robot, this would pose a threat to the burr material, as well as to the patient. After implementation of sensory feedback to control the robot speed inversely proportional to a function of the averaged absolute forces, we observed a great difference; the robot's movements were much smoother, resembling the manner in which a surgeon would fulfill the task. When encountering low resistance, the robot moves quickly, whereas it moves slowly when registering high force values. The force levels neither surpassed the limit nor caused abortion of the task. As the forces were averaged over a sample of seven measurements to control the robot task in force feedback, a single peak above the limit did not cause abortion of the task, but only the averaged force level greater than $10 \mathrm{~N}$. The robot was then able to mill an exact implant bed (Fig. 10A) for the main module of the cochlear implant by Med-El, Inc. (Fig. 10B), or the totally implantable hearing system TICA.

\section{DISCUSSION}

The current registration method is simple. The problems encountered should be solvable by implementing ultrasound-guided local navigation. This may be later combined with global navigation using an already commercially available infrared-based system. The paths for each implant can be calculated online. For the individual patient, these paths are positioned and orientated intraoperatively after registration of the robot to the patient. It is of great importance to avoid heat or mechanical trauma. ${ }^{6}$ One effective way to achieve this goal proved to be the force-based speed control implemented in our system. ${ }^{7}$ Another important factor, namely the elimination of tall grooves in the cavity floor, is achieved by choosing a small distance between paths. Because the resulting increase in total procedure time is relatively small, a good compromise is to halve the radius of the cutting burr used.

Even when the robot was motionless and the burr was not running, the SD of forces successively collected for a set of 20 measurements did not equal $0 \mathrm{~N}$. If the robot
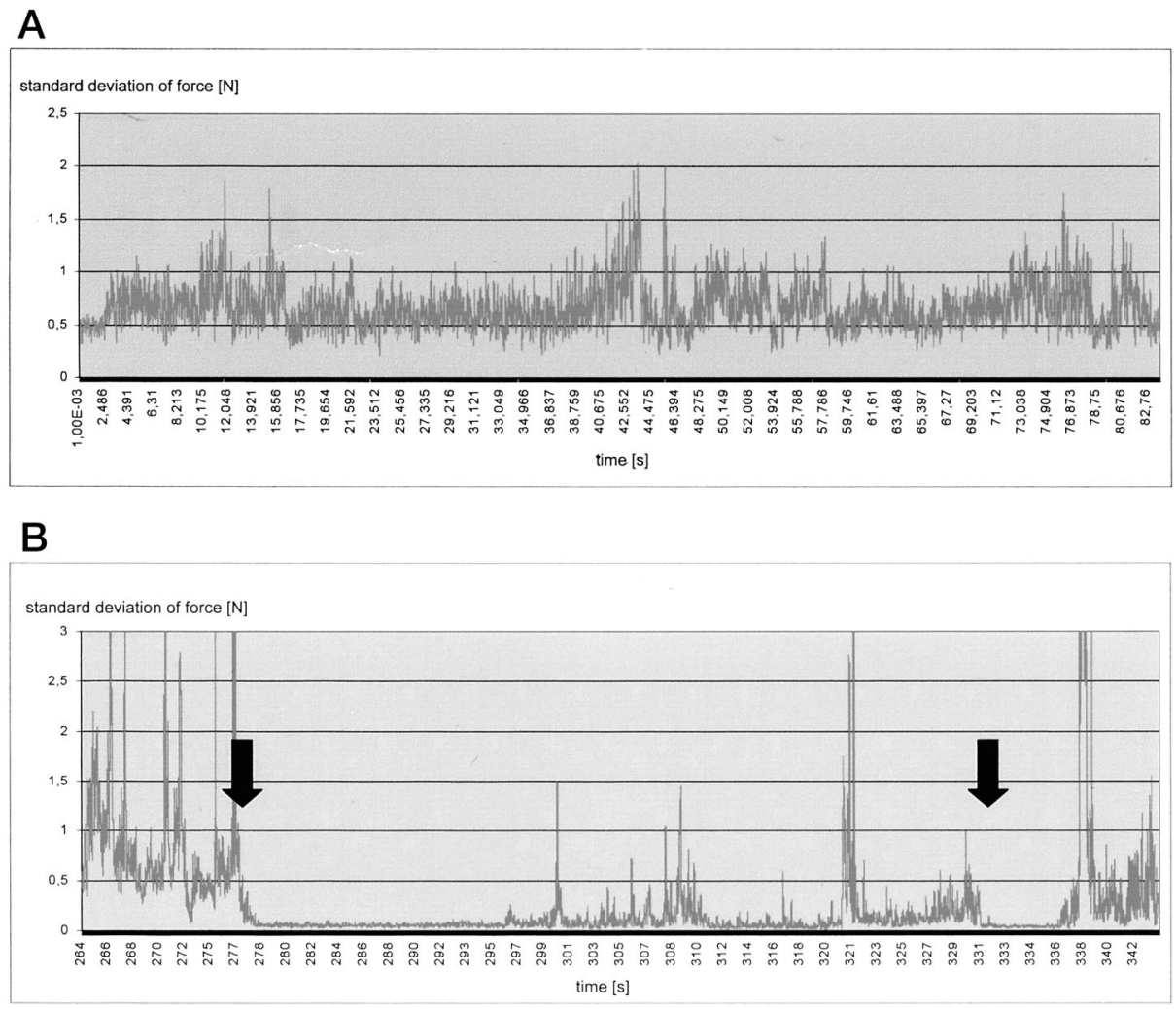

Fig. 8. (A) Standard deviation of force measured during milling in the temporal bone specimen (mean values of a sample of 20 measurements). (B) Standard deviation of force measured during milling in the temporal bone specimen (mean values of a sample of 20 measurements). The drop is evident when the dura mater is reached at times 227.5 and 332 seconds (marked by arrows). 


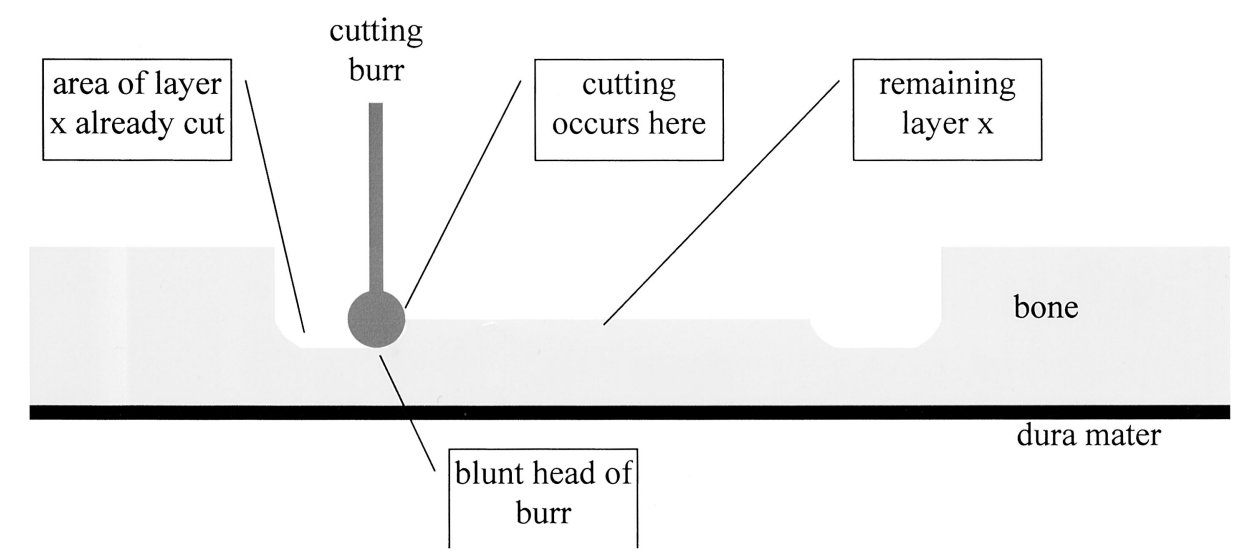

Fig. 9. Sketch of the spiral horizontal milling mode. Cutting occurs at the sharp side of the cutting burr but not at the rather blunt head of the burr. was not moving, the force SD increased only slightly because of vibration when the burr was operating. It was interesting to observe that the force SD oscillated to a high extent when the robot was moving and the burr was active. This phenomenon may be due to the vibrations caused by the burr, which are also measured by the force sensor because they transmit to the bone through the tight coupling. When this hard, bony layer is gone and the burr has reached the dura, the soft tissue absorbs vibrations and damps the SD. This drop is dramatic and therefore can easily be automatically recognized by the robot. Occasional peaks observed are probably due to tiny remnants of bone on the dura.

Even more surprising was the fact that the cutting burr could be observed to move on the dura for long periods (i.e., several planes [!]) without causing any dural damage. This is due to the manner in which the robot holds the cutting burr, that is, always perpendicular to the surface (Fig. 9); in contrast, a surgeon deliberately tilts the burr to work with the sharp edges. Therefore, only the blunt top of the burr head comes into contact with the dura during robotic milling because removal of the bone occurs laterally in the horizontal milling mode (Fig. 9) and only at one point when changing depth (i.e., when moving to the next plane underneath). Moreover, this vertical movement $r_{4}$ by the robot is variable and was set to $1 \mathrm{~mm}$.

The implementation of inversely proportional sensory feedback to control the robot speed was a great step forward, avoiding subjecting the specimen (and in the future, the patient) to forces greater than the allowable limit. This was intended to be simply a safety measure, but as an additional benefit the movements of the robot became much more human-like. Just as a surgeon would reduce his or her cutting pressure (but not the burr's rotation speed) when encountering a section of bone with high cutting resistance, so does the robot at present. Of course, the burr's rotation has to be kept fast enough to effectively reduce resistance. The resistance depends on the actual speed of the cutting edges as a function of revolving speed and diameter of the burr head. Ample cooling by the integrated saline irrigation system is a prerequisite, as well as thorough suctioning of bone material cut away by the burr. ${ }^{5}$ It the end, the main module of the cochlear implant by Med-El, Inc., or the totally implantable hearing system TICA could be easily placed into the cavity (Fig. 10). Although today these prosthetics can be implanted without the aid of robotics, the robot spares the surgeon from spending time on and devoting attention to a coarse procedure before the microsurgery (i.e., placement of the electrode or the transducer). In the near future, more electronic implants may be introduced into head and neck surgery or otoneurosurgery, increasing the number of procedures that could benefit from robotic precision.

\section{CONCLUSION}

The study reports the first development of an in vitro functional robotic milling system for creating cavities for implants in otoneurosurgery, such as cochlear implants or implantable hearing devices. Because of the
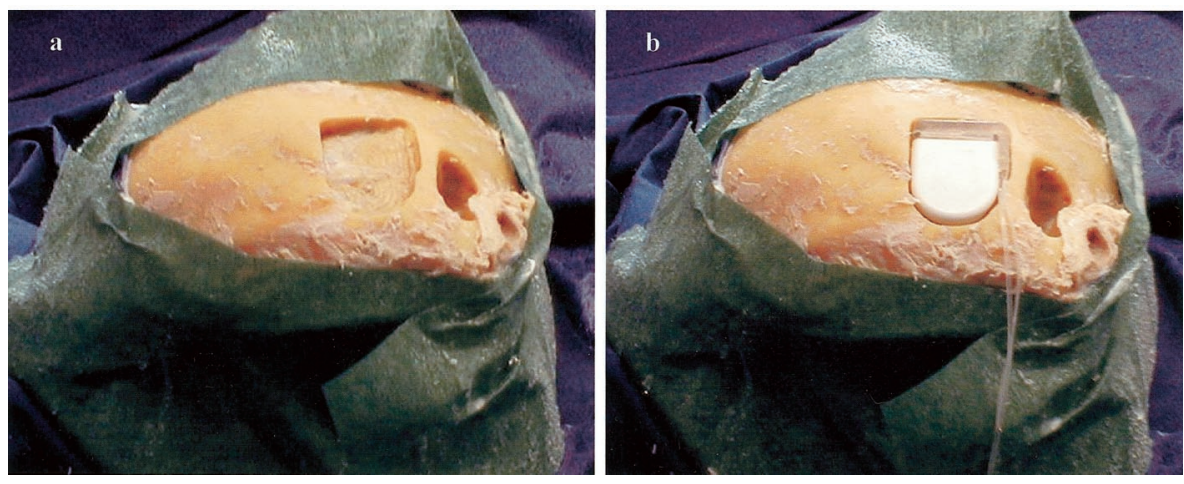

Fig. 10. Right-side human temporal bone specimen fixed in formaldehyde (with consent of body donor and approval by local ethics commission). (A) Implant cavity milled out by the robot in the spiral horizontal mode with a $4.5-\mathrm{mm}$ cutting burr. The mastoid cavity was created manually with the cutting burr. (B) Cochlear implant by Med-El, Inc., placed into the cavity. 
force-based velocity control, the robot moves according to the encountered resistances in an almost human-like fashion. Moreover, the robot can automatically detect the dura mater without damaging it by analyzing the $\mathrm{SD}$ of the forces encountered. Future plans include implementation of an ultrasound-based sensor to enable local navigation by measuring the outer and inner contours of the calvarial bone before the milling task. However, the robotic milling of an implant bed is only the first step in our scientific program, which includes the realization of robotic mastoidectomy. To achieve this, further work has to performed to implement other safety features, such as neuromonitoring of the facial nerve and detection of the sigmoid sinus. This step may be combined with global navigation using an infraredbased system and planning off-line based on computer tomography data. Furthermore, minimally invasive surgery to the lateral base of the skull may be possible with the robot.

\section{BIBLIOGRAPHY}

1. Davies B. A review of robotic surgery. Proceedings of the Institution of Mechanical Engineers. Part H, Journal of Engineering in Medicine 2000;214:129-140.

2. Federspil PA, Stallkamp J, Plinkert PK. Robotik: Ein Evolutionssprung in der operativen Medizin? Deutsches Ärzteblatt 2001;98A:2879-2884

3. Federspil PA, Stallkamp J, Plinkert PK. Robotik: Eine neue Dimension in der HNO-Heilkunde? HNO 2001;49:505-513.

4. Plinkert PK, Plinkert B. Robotics in skull base surgery. Proceedings of the 15th International Congress CARS. Amsterdam: Elsevier Science, 2001:139-144.

5. Plinkert PK, Plinkert B, Hiller A, Stallkamp J. Einsatz eines Roboters an der lateralen Schädelbasis: Evaluation einer robotergesteuerten Mastoidektomie am anatomischen Präparat. HNO 2001;49:514-522.

6. Fuchsberger A. Untersuchung der spanenden Bearbeitung von Knochen. Forschungbericht iwb. Berlin: Springer, 1986.

7. Henrich D, Plinkert PK, Federspil PA, Plinkert B. Robotergestütztes Fräsen an der lateralen Schädelbasis: Kraftbasierte lokale Navigation bei der Implantatbettanlage. VDI-Bericht 1679-Tagungshandbuch zur Robotik 2002, Ludwigsburg, 2002. VDI Verlag, Düsseldorf, pp 497-502. 